\title{
Diversity and distribution of the genus Arachniodes Blume (Dryopteridaceae) in North-East India
}

\author{
A. Benniamin ${ }^{1}$ and M. Shunmuga Sundari ${ }^{2}$ \\ ${ }^{1}$ Corresponding author: Botanical Survey of India, Western Regional Centre, Pune 411001, Maharashtra, \\ India.E-mail: fernsbenni@gmail.com \\ ${ }^{2}$ Department of Botany, S.P. Pune University, Pune 411001, Maharashtra, India
}

[Received 03.08.2019; Revised 13.12.2019; Accepted 17.12.2019; Published 31.12.2019]

\begin{abstract}
The present study is mainly based on the field work conducted from 2008-2013 to different parts of North East India. Altogether six species of Arachniodes Blume collected from North East India which Arachniodes miqueliana (Maximowicz ex Franchet \& Savatier) Ohwi. is collected for the first time from India and it is reported as new record for India. Detailed key for all the species, descriptions and photos for easy identification. All the collected species have been deposited at Botanical Survey of India, Western Regional Centre, Pune. Detailed description, key for each species, GIS mapping for each and every species and Photographs are also provided for easy identification.
\end{abstract}

Key words: Biodiversity, Conservation, Pteridophytes, Dryopteridaceae

\section{INTRODUCTION}

The North-East region has been under focus for its high biodiversity and the region has been a priority for investment by the leading conservation agencies of the world. The region's lowland and montane moist to wet tropical forests are considered to be the northernmost limit of true tropical rainforest in the world (Proctor et al. 1998). The ferns originated in ancient tropical habitats and have been successful in many types of environments for thousands of millennia. According to Rothwell and Stockey (2008) ferns first appeared in the fossil record during the Middle Devonian 390 million years ago. Ferns have continued to evolve and diversify up to the recent ages. However, according to Chapman (2006) about $15 \%$ of all fern and lycophyte species may not yet be known to science. The total number of known ferns is estimated at about 12,000 species worldwide belonging to 225-230 genera (Kramer 1990) and 37 families (Smith et al. 2006).

Dryopteridaceae is of cosmopolitan distribution although with a strong concentration in the temperate regions of the Northern Hemisphere, particularly in the hills and mountains of eastern Asia. The Family was known only by 134 taxa under 15 genera from India. So far, 12 genera have been reported in North East India. The genus Arachniodes Blume is consisted to have 50-70 species distributed in tropical, subtropical and southern temperate regions of the world (Kramer et al. 1990). In India 12 species have been reported by Chandra (2000). But several workers have done lot of works in North East India. Though several regional revisions were carried out in last decades (on Azolla and Salvinia, Griffith 1845; Cryptogamsic plants, Roxburgh 1844; Hooker (Species Filicum Vol 1-5, 1844 - 1864); Beddome (Ferns of Brit Ind.1865-1870, Suppl. Ferns Brit. India 1876; Handb.1883; with suppl.1892; New ferns 
collected from Manipur, 1888); Clarke (A review of ferns of Northern India, 1880 and On the ferns of Nagaland and Manipur, 1888); Hooker and Baker (Synopsis Filicum, 1864,1874); Clarke and Baker Notes on ferns of Northern India, 1888), Burkill (the botany of Abor Exped., 1924); Fisher (The ferns of Lushai Hills, 1938); Kachroo (Ferns of Asssam, 1975); Panigraghi (Pteridophytes of Eastern India, 1962); Panigraghi and Patnaik (Enumeration of correct nomenclature of Polypodiaceae in Eastern India, 1961), Panigraghi and Chowdhury (Enumeration and distribution of fern allies in Eastern India, 1962). Widén et al. (1996), with 24 species of those genera being noted as occurring in Arunachal Pradesh. Vasudeva et al. (1990) compiled a list of 73 species of Dryopteridaceae from North Eastern India, but few records were known to them from Arunachal Pradesh. Biswas (1941) reported 4 species collected by Bor in Kameng, Srinivasan (1959) reported 5 species from Kameng, Chandra (1980) reported 19 species from Kameng, Rao and Hajra (1980) reported 32 species from Kameng, Deb and Dutta (1972) reported 4 species from Tirap; Chauhan and Wadhwa (1996) reported 7 species from Tirap; Singh and Panigrahi (2005) recorded 15 species from Tirap district and Rawat et al. (2009) reported 21 species from Mehao Wildlife Sanctuary, Lower Dibang Valley. The main aim of study is to bring out a detailed account on the genus Arachniodes Blume from North East India.

\section{METHODS}

The present work is based on extensive collections made regularly from several areas in different parts of North East India. These areas were visited periodically in different season during 2008-2013. In presenting the account here, the classification followed here in basically according to Pichi-Sermolli's (1977) with minor changes following Fraser-Jenkins (2010). The taxonomic treatment includes explicit keys to genera and species under family along with detailed description for each species. In general, the descriptions of species were made from either herbarium of from live specimens and similarly species under general are also arranged alphabetically. In determining name of taxa, an effort has been made to present, as far as possible, the nomenclature accepted as valid in current literature. In this connection all the literature available to the authors are consulted. Only important synonyms which have relevance to Indian works. Original citation of both accepted name and synonyms are provided. Distribution Map and Colored Photograph also provided for easy identification.

\section{Key to the 12 Genera known to occur in North-eastern India}

1a. Swollen base of stipe bearing glossy scales; axes of frond bearing short unicellular hairs only - - - - Hypodematium

1b. Base of stipe not swollen. scales usually on other parts also hairs on axes of fronds mostly not unicellular - . . . . . . - 2

2a. Rhizome usually creeping, frond approximate - . - - - Arachniodes

2b. Rhizome erect, short suberect, frond tufted - - - - - - - - - - - - - - - 3

3a. Costae and costules densely scaly in abaxial surface - - - - - - - - Ctenitis

3b. Ctenitis hairs are absent

4a. Veins anastomising -

4b. Veins others -

5a. First pinnule of all pinaae except the lowest anadrmous, rachis uslally more or less zig zag - Acrorumohra

5b. First pinnule of all pinnae except the lowest catadrmous - - - - - - - - 6

6a. Lamina with a tooth in each sinus, the teeth elevated on adaxial surface - - - Pleocnemia 
6b. Lamina margin without any teeth - - - - - - - - - 7

7a. Stipte and rachis purple, lamina bear intesitiniform hair like setae - - - - - Nothoperenema

7b. Stipe and rachis brown or straw coloured, intesitiniform setae absent - - -8

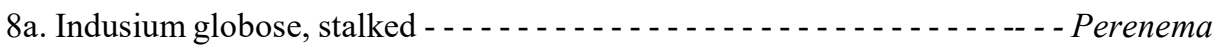

8b. Indusium reniform - - - - - - -

9a. Veins anastomosing, scattered sori - - - - - - - Cyrtomium

9b.Veins free - - - - - - 10

10a. Pinnules with stiff, aristae margin; indusial peltate, centrally attached - - Polystichum

10b. Pinnules without stiff - - _ - _ - 11

11a. Indusium reniform attached by a sinus, margin of pinnule serrate proliferating buds absent - - - - - - - - - - - - - - - Dryopteris

11b. Indusium not as above

\section{TAXONOMIC TREATMENT OF THE GENUS Arachniodes Blume}

\section{Key to the Species}

1a. Rhizome with dense tuft brown scales - - - - - - - - - - Arachniodes superba

1b. Rhizome otherwise - - - - - - -

2a. Lamina rigescent, Sori terminal on veinlets -

2b. Lamina soft, shinning green, Sori on veinlets - - - - - Arachniodes henryii

3a. Fronds 4 pinnate in basal part - - - - $-1 .-4$

3b. Fronds 5 pinnate in basal part -

4a. Veins abaxially scattered with brown bullate basal scales - - - - - Arachniodes miqueliana

4b. Veins abaxially not scattered without scales - - - - - - - - - 5

5a. Rhizome long creeping, ultimate pinnule $1.5 \mathrm{~cm}$ long - - - - - - Arachniodes rhomboidea

5b. Rhizome short creeping, ultimate pinnule 3.5cm long - - - - - Arachniodes assamica

Arachniodes assamica (Kuhn) Ching, Acta Bot. Sin. 10(3): 256. 1962; Ohwi, J. Jap. Bot. 37: 76. 1962; N.C. Nair et Chandra, Bull. Bot. Surv. India 11(1\&2): 185. 1969; Aspidium assamicum Kuhn, Linnaea 36: 108. 1869. Aspidium aristatum var. assamicum (Kuhn) Baker, in Hooker \& Baker, Syn. Fil., ed.2, 493. 1874. Polystichum aristatum var. assamicum (Kuhn) C.Chr., Index Fil. 579. 1906. Dryopteris assamica (Kuhn) Rosenst., Med. Rijks Herb. 31: 6. 1917. Dryopteris speciosa var. assamica (Kuhn) C.Chr., Contrib. U. S. Natl. Herb. 26(6): 282. 1931. Rumohra assamica (Kuhn) Ching, Sinensia 5(1-2): 47-49, t. 6. 1934. Arachniodes assamica (Kuhn) Ching, Act. Bot. Sin. 10(3): 256. 1962, Aspidium yoshinagae Makino, Bot. Mag., Tokyo 13: 57. 1899. Polystichum yoshinagae (Makino) Makino, Bot. Mag., Tokyo 23: 144. 1909. Arachniodes yoshinagae (Makino) Ching, Act. Phytotax. Sin. 9: 383. 1964. Rumohra assamica forma simplicipinnata Ching, Sinensia 5(1-2): 49. 1934.

Rhizome short creeping, Stipe up to $45 \mathrm{~cm}$ long, scaly on lower part, scales brown, lanceolate, acuminate, entire, sparsely fibrillose upwards. Lamina lamina deltoid, bipinnate or tripinnatifid glabrous, lustrous green, thin, chartaceous simple pinnate form; pinnae 6-8 pairs, lanceolate acuminate inciso-cuspidate, stalked under pinnatifid apex, in bipinnate or tripinnatifid form; acuminate, lateral pinnae 4-6 pairs, stalk long, oblique, basal pinnae over 20 $\mathrm{cm}$ long, pinnate with about 10 pairs of the deltoid lanceolate, oblique pinnules, unequally 

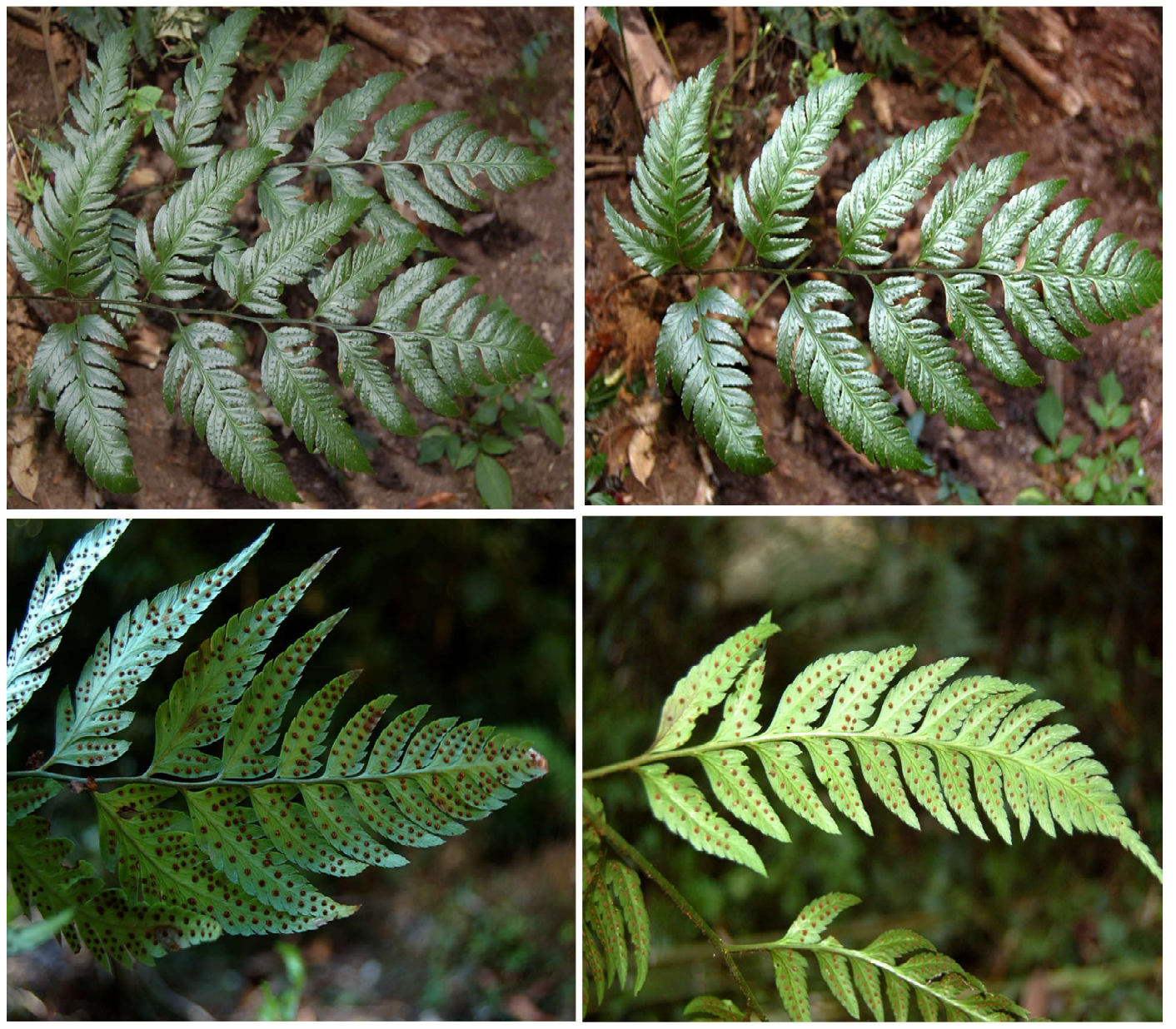

Figure 1. Arachniodes assamica (Kuhn) Ohwi
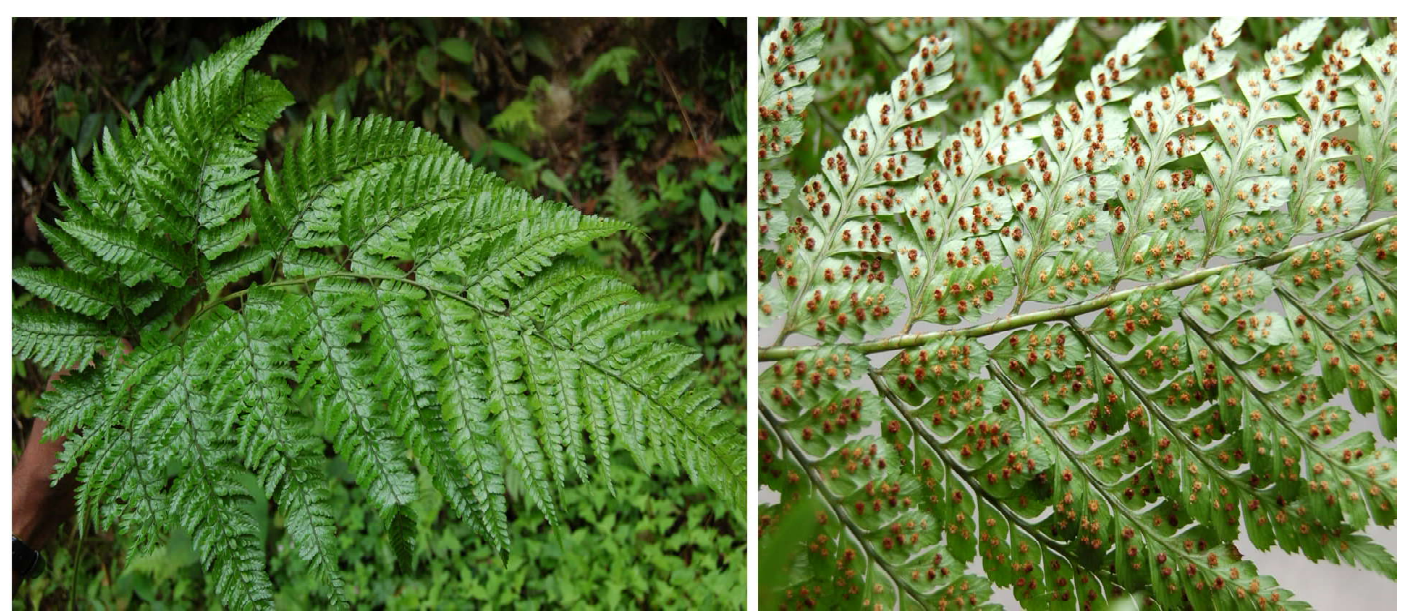

Figure 2. Arachniodes ciniifolia (T.Moore) Ching 
lobed, 4-6 cm long, 1-1.5 cm broad, obliquely truncate, and slightly auriculate on anterior side, cuneate below, margin incised half way down or serrate with about 4-b7 large teeth with mucronate point; veins and veinlets, anterior basal veinlet of each group terminated by a large rounded sorus some distances below the sinus; sori uniseriate, indusium large, reddish, entire, round-reniform, (Figure 1; Map 1)

Ecology: It grows on forest floor from 1500 to $2000 \mathrm{~m}$ amsl.

Distribution: INDIA: North-east India: (Assam, Manipur), Sikkim, West Bengal, CHINA, JAPAN, MYANMAR.

Specimen examined: Arunachal Pradesh: W. Siang, Mechuka, A.Benniamin 25294, dated November 2008, XCH; Tirap, Langsang Forest, D.B. Deb 26172, dated 26.06.1961, ARUN.

Arachniodes coniifolia (T. Moore) Ching, Acta Bot. Sin.10(3): 257. 1962; N.C. Nair, Bull. Bot. Surv. India 11(1-2): 186. 1969; Lastrea coniifolia T. Moore, Ind. Fil. 1xxxviii. 1857.; Bedd., Ferns Brit. Ind. t. 261. 1868; Handb.230. T. 118. 1883; Suppl. 50. 1892. Rumohra wallichii (D. Don) Ching, Sinensia 5: 56, pl. 9. 1934. Aspidium coniifolium Wall. ex Kunze in Linnaea 24: 293. 1891; non C. Presl, 1822; Lowe, Ferns Brit. \& Exot. 6. t. 39. 1871. Nephrodium coniifolium C.Hope, J. Bombay Nat. Hist. Soc. 14: 720. 1903(in part). Lastrea aristata var. coniifolia T. Morre, Ind. Fil. 88. 1858. Aspidium aristatum var. coniifolium Hook., Sp. Fil. 4: 28. 1862; Syn. Fil. 255. 1867.

Rhizome erect, stipe tufted, rhizome and base of stipe densely scaly; rhizome scales ovate-lanceolate, long acuminate, margin fimbriate apex, stipe stramineous, up to $65 \mathrm{~cm}$ long; lamina 5-pinnate, large, ovate, up to $100 \mathrm{~cm}$ long, pinnae up to $40 \mathrm{~cm}$ long, ovate lanceolate, upper pinnae oblique enlarged towards basioscopic side, other pinnae $15 \mathrm{~cm}$ broad, second pinnae $25 \mathrm{~cm}$ long, $10 \mathrm{~cm}$ broad, oblique, 15 pairs, apex long acuminate, tertiary pinnae up to $6 \mathrm{~cm}$ long, $2 \mathrm{~cm}$ broad, pinnae of the $4^{\text {th }}$ order $1.5 \mathrm{~cm}$ long, $0.5 \mathrm{~cm}$ broad, rhomboid, pinnae lobed at base; lobation reach up to midrib, ultimate segments, small, $5 \mathrm{~mm}$ long, $2 \mathrm{~mm}$ broad, margin at upper portion with 4-5 arista. Sori large, terminal on veinlets, indusium round-reniform. (Figure 2; Map -1)

Ecology: It grows on forest floor from 1000 to $2500 \mathrm{~m}$ amsl.

Distribution: INDIA: North East India: (Arunachal Pradesh, Manipur, Meghalaya), Sikkim, Peninsular India, BHUTAN, CHINA, NEPAL.

Specimen Examined: Arunachal Pradesh: Upper Subansiri, Daporijo, A.Benniamin 22787, dated 10.06.2008, ARUN; Kurungkumey, Serley [Sarli], A.Benniamin 26459, dated December 2008. Nagaland: Mokochungdist, Changkiforest area, A.Benniamin, 2835023, dated July.2011, ARUN; Manipur: Sennapathi dist, Jackamma hills, A.Benniamin 26845, dated 18.02.2010, ARUN. Sheroy hills, A.Benniamin, 26743, 26729, dated 14.02.2011 ARUN; Nagaland: Tuensang dist, Nongchugang forest area, A.Benniamin 28388, 23.07.2011, ARUN.

Arachniodes henryi (Christ) Ching, Acta Bot. Sin. 10(3): 258. 1962. Polystichum henryi Christ in Lecomte, Not. Syst. 1: 36. 1909; Rumohra henryi (Christ) Ching, Sinensia 5: 57, pl. 10. 1934. Byrsopteris henryi (Christ) C.V.Morton in Am. Fern J. 50: 154. 1960. Dryopteris henryi (Christ). C. Chr., Contr. U.S. Nat. Herb. 26:282. 1930.

Rhizome long creeping, stipe distant, rhizome thick a finger and $1 \mathrm{~cm}$ dia., rhizome and base of stipe densely covered with subulate-lanceolate, 5-6 mm long, blackish brown undulate, 

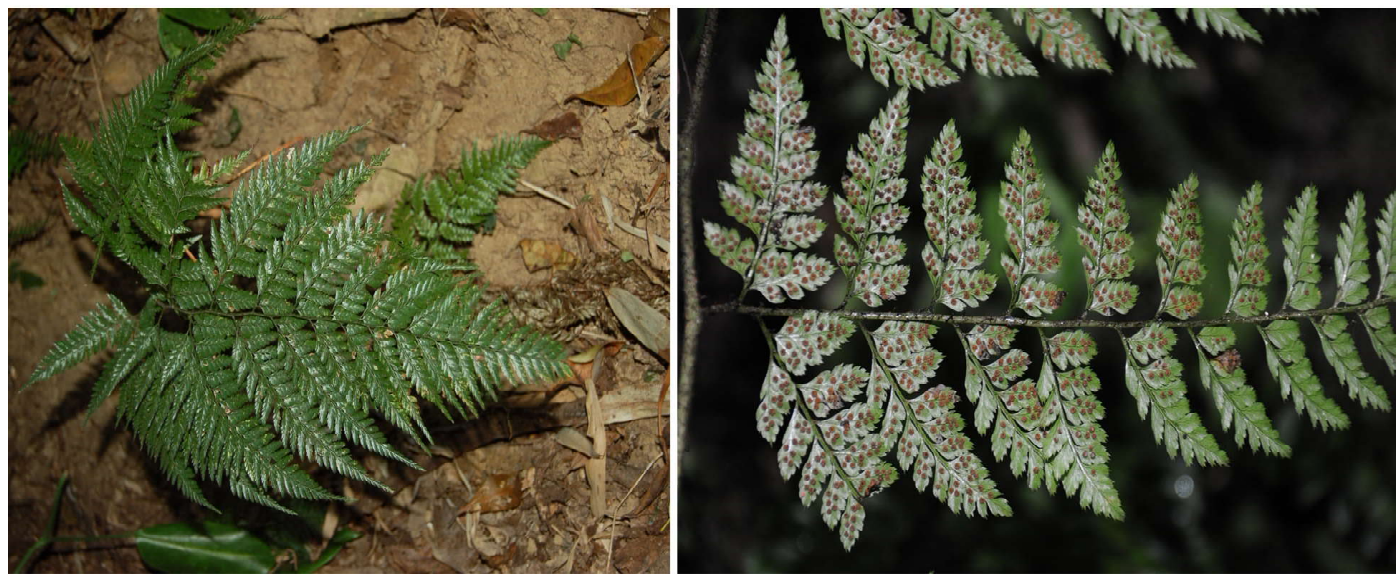

Figure 3. Arachniodes henryi (Christ) Ching
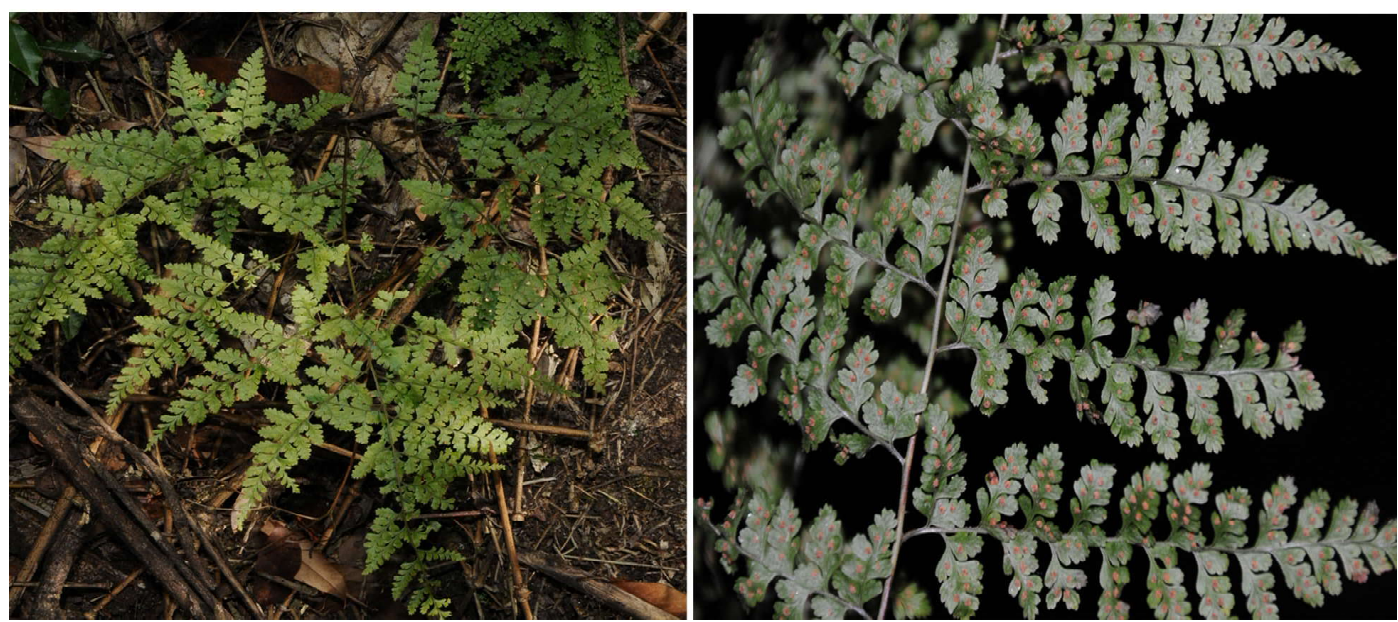

Figure 4. Arachniodes miqueliana (Maxim. ex Franch \& Sav.) Ohwi
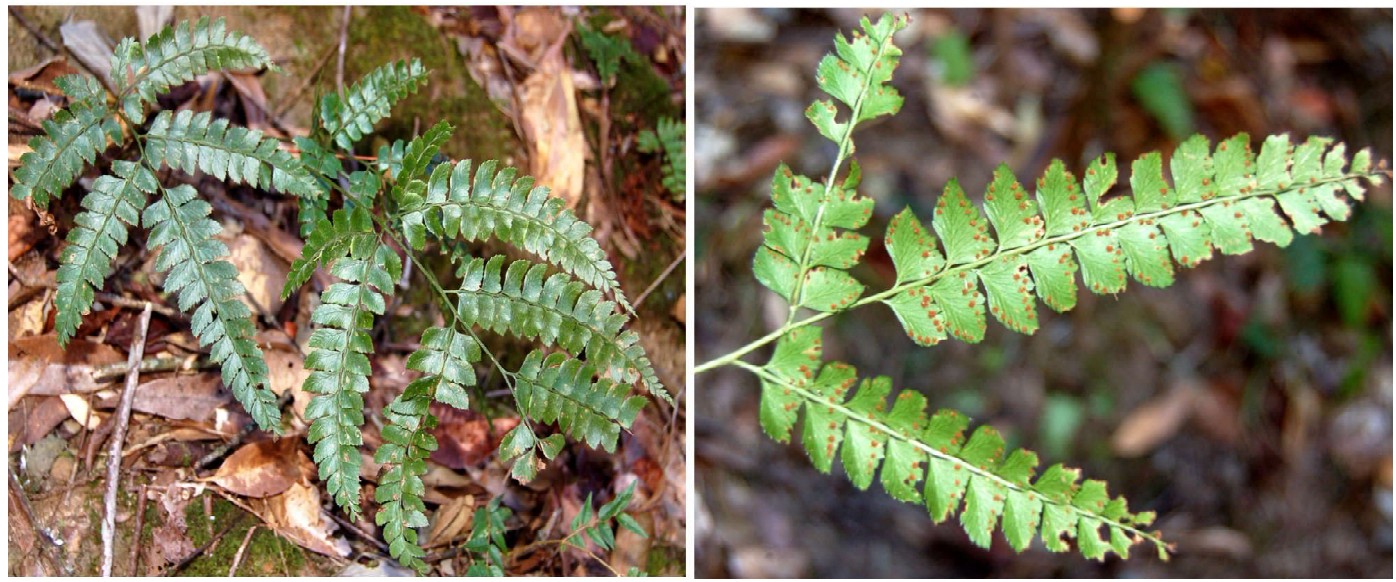

Figure 5. Arachniodes rhomboidea (Wall.) Ching 
subadpressed scales, stipe scales 7-5 mm long, lanceolate subulate, brown, stipe up to $55 \mathrm{~cm}$ long, brownish; lamina 4-5 pinnate at base pinnate above, soft, shining green, large up to 50 $\mathrm{cm}$ or more long, broadly deltoid, primary pinnae 13 pairs, stalked, remote lowest pinnae largest, about $25 \mathrm{~cm}$ long, deltoid oblong, acuminate; secondary pinnae 12 pairs, stalked, elongate-acuminate, basal secondary pinnae largest, $12 \mathrm{~cm}$ long, $5 \mathrm{~cm}$ broad, acute, tertiary pinnae acute up to $3 \mathrm{~cm}$ long, deltoid; ultimate segments $1 \mathrm{~cm}$ long, $5 \mathrm{~mm}$ broad, inciso serrate, lobe aristate, dentate; Sori dorsal on veinlets closer to costule than the margin, Indusium reniform, brown, undulate (Figure 3; Map-1).

Ecology: It grows under shade from 1200 to $2000 \mathrm{~m}$ amsl.

Distribution: INDIA: Eastern India (Meghalaya, Manipur, Mizoram, Nagaland), Nepal, China

Specimen examined: Arunachal Pradesh: Kurungkumey, A.Benniamin s.n., ARUN; Siang, Mechuka, A.Benniamin XCH s.n. 25113. 25330, dated 18.11.2008, ARUN.

Arachniodes miqueliana (Maximowicz ex Franchet \& Savatier) Ohwi, J. Jap. Bot. 37: 76. 1962. Aspidium miquelianum Maxim. ex Franch. \& Sav., Enum. Pl. Jap. 2: 240. 1876. Nephrodium miquelianum (Maxim. ex Franch. \& Sav.) Kom., Fl. Manchur. 1: 123. 1901. Dryopteris miqueliana (Maxim. ex Franch. \& Sav.) C.Chr., Index Fil. 278. 1905. Rumohra miqueliana (Maxim. ex Franch. \& Sav.) Ching, Sinensia 5: 67, t. 17. 1934. Leptorumohra miqueliana (Maxim. ex Franch. \& Sav.) H.Itô, in Nakai \& Honda, Nov. Fl. Jap. 4: 119. 1939. Polystichopsis miqueliana (Maxim. ex Franch. \& Sav.) Tagawa, J. Jap. Bot. 33: 94. 1958. Aspidium fargesii Christ, Bull. Soc. Bot. France 52, Mém. 1: 42. 1905. Dryopteris fargesii (Christ) C.Chr., Index Fil. 264. 1905. Leptorumohra fargesii (Christ) Nakaike \& A.Yamam., J. Nat. Hist. Mus. Inst. Chiba 3(2): 144. 1995. Arachniodes borealis Seriz., J. Jap. Bot. 61(2): 48. 1986.

Rhizome long creeping, densely clothed with brown lanceolate scales along growing parts. Frond $0.4-1.3 \mathrm{~m}$; stipes $25 \times 54 \mathrm{~cm}$ and $3-5 \mathrm{~mm}$ in diam., brownish stramineous, copiously scaly throughout primary rachis; lamina herbaceous, obscurely green to yellowish green when dried, deltoid-pentagonal, $35-52$ x 26-35 cm, apex acuminate, 4-pinnate; pinnae 6-10 pairs, alternate, obliquely spreading, lowest pinna deltoid-ovate, $29-32 \times 22-25 \mathrm{~cm}$, apex acuminate, 3-pinnate; first order pinnules 18 pairs, obliquely spreading, deltoidlanceolate, basal basiscopic one $15 \times 7 \mathrm{~cm}, 2$-pinnate; penultimate pinnules 6-7 pairs, stalked, blunt deltoid to oblong, $c a .3 .5 \times 2.5 \mathrm{~cm}$, base broadly cuneate, apex obtuse, 1pinnate; ultimate pinnules 3-4 pairs, ovate or rhombic, to $1.2 \times 0.9 \mathrm{~cm}$, apex obtuse or acute, margin deeply pinnatifid or obtusely serrate; upper pinnae gradually reduced; axes and main veins adaxially with pale grey unicellular acicular hairs, veins abaxially scattered with brown, ovate scales. Sori 1-3 per ultimate segment; indusia brown, entire. (Figure 4; Map 1).

Ecology: Under broad-leaved or mixed forests, on earth or occasionally on shaded moist and moss covered rock surface at $1200-1450 \mathrm{~m}$ amsl.

Distribution: INDIA: North East India: (Arunachal Pradesh), JAPAN, KOREA.

Specimen examined: Arunachal Pradesh: Kameng, Jang, Tawang, A.Benniamin 28050, 2010, dated 10.08.2010, ARUN.

Arachniodes rhomboidea ((Wall. ex C.Presl) Ching, Act. Phytotax. Sin. 9(4): 383 - 384. 1964. Polystichum rhomboideum Schott, Gen. Fil. t. 9. 1834. Aspidium rhomboideum (Wall. ex C.Presl) Mett., Abhandl. Seckenb. Naturf. Ges. 2(4): 350. 1857. Polystichum 

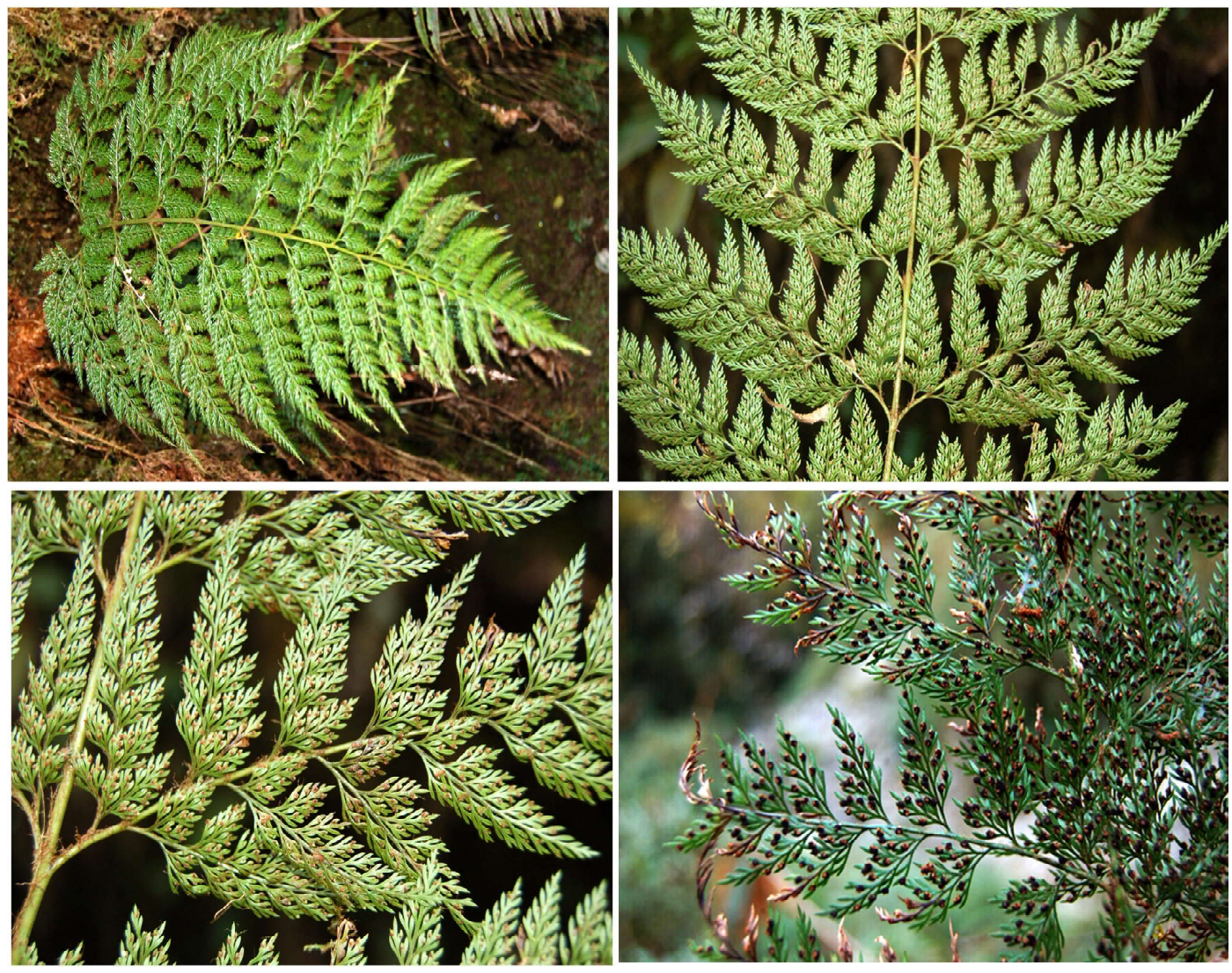

Figure 6. Arachniodes superba Fraser-Jenk.

controversum (Hance) Ching, Coll. Dept. Biol. Coll. Sci. Sun YatsenUniv. 6: 7. 1933. Byrsopteris amabilis var. yakusimensis (H.Itô) Sa.Kurata, in Namegata \& Kurata,Enum. Jap. Pterid. 283, 339. 1961. Arachniodes amabilis var. yakusimensis (H.Itô) Ohwi, J. Jap. Bot. 37: 76. 1962. Arachniodes rhomboidea var. yakusimensis (H.Itô) W.C.Shieh, Fl. Taiwan 1: 365. 1975. Arachniodes yakusimensis (H.Itô) Nakaike, New Fl. Japan Pterid. 839. 1992. Arachniodes amabilis var. fimbriata K.Iwats., Act. Phytotax. Geobot. 30(1-3): 42.1979.

Rhizome long creeping, densely scaly, scales brown, stipe $30-40 \mathrm{~cm}$ long, brownish, slender; lamina 30-50 cm long, bipinnate, subcoriaceous, ovate oblong, pinnae 5-12 pairs, lateral pinnae similar, pinnae up to $15 \mathrm{~cm}$ long, $3.5 \mathrm{~cm}$ broad, all pinnae stalked, basal basioscopic pinnules of the lowest pinnae again pinnate, pinnules stalked, rhomboid, $1.5 \mathrm{~cm}$ long, subfalcate, acute subauriculate or none, margin spinulose serrated except lower base. Sori in single row at sinus of the serrate, close to the margin, terminal. Indusium reniform, fringed. (Figure 5; Map 1).

Ecology: It grows from 1000 to 1200 m altitude in semi shade forest area.

Distribution: INDIA: North East India: (Mizoram, Meghalaya), CHINA, NEPAL, TAIWAN.

Specimen examined: Mizoram: Murlen National Park, A.Benniamin, 22083, 2012, ARUN. 
Arachniodes superba Fraser-Jenk., New Sp. Syndr. Indian Pteridol. 39. 1997. Aspidium foeniculaceum Hook., Sp. Fil. 4: 36, t. 237. 1862. Lastrea foeniculacea (Hook.) Bedd., Ferns Brit. India t. 36. 1865. Polystichum foeniculaceum (Hook.) J.Sm., Hist. Fil. 220. 1875. Diacalpe foeniculacea (Hook.) C.B.Clarke, Trans. Linn. Soc. London, 2 Bot., 1: 434. 1880. Dryopteris foeniculacea (Hook.) Kuntze, Revis. Gen. P1. 2: 812. 1891. Lithostegia foeniculacea (Hook.) Ching, Sinensia 4: 2. 1933. Peranema foeniculacea (Hook.) B.K.Nayar \& S.Kaur ex S.Chandra \& S.Kaur, Nomenc. Guide Beddome's Ferns S. Brit. India 38. 1987.

Rhizome short oblique, stout, densely scaly, stipe tufted, $18-40 \mathrm{~cm}$ long or more, rufo stramenous, angular below, scaly scales dense, $1 \mathrm{~cm}$ long, brown, lanceolate, long acuminate, margin more or less fimbriate; lamina large, $45-80 \mathrm{~cm}$ long, $30-50 \mathrm{~cm}$ broad, deltoid acuminate, 4-5 pinnate with very ultimate division; Primary pinnae about 14pairs, alternate, stalked, oblique, basal pairs largest, $18-30 \mathrm{~cm}$ long, $15-25 \mathrm{~cm}$ broad, deltoid, acuminate, anadromous, rachilets fibrillose scaly; secondary pinnae oblong lanceolate, up to $10 \mathrm{~cm}$ long, $3-4 \mathrm{~cm}$ broad, petiolulate, oblique, pinnae of third order shortly petiolulate, $2.2-5 \mathrm{~cm}$ long, $1-1.5 \mathrm{~cm}$ broad, base unequal, oblong ovate, oblique, pinnae of the $4^{\text {th }}$ order, $1 \mathrm{~cm}$ long, $6 \mathrm{~mm}$ broad, pinnate, up to 1-3 pairs, oblique, linear, sharply pointed, ultimate segments, 2-3 mm long, 1-1.5 mm broad, entire, provided with one simple veinlets, veinlets ended far below apex,texture softly subcoriaceous, under side of costules, veins and ultimate segments with twisted, brown, soft, long hair like fibrillose scales, clavate tip of veinlets prominent on the upper side of segment; sori small, rounded, indusiate, one to each segments; indusium thick, coriaceous, or almost dark purple, ovate, oblong, entire, covering the entire sori at first, splitting into 2-3 turned aside, subrounded lobes with exterior connate lateral side is persistently attached to receptacle (Figure 6; Map 1).

Ecology: It grow as terrestrial between 1400 and $1880 \mathrm{~m}$ amsl.

Distribution: INDIA: North East India (Arunachal Pradesh), Sikkim, West Bengal, CHINA Specimen examined: Arunachal Pradesh: Mechuka, A.Benniamin 26094, dated November 2008, ARUN.

\section{DISCUSSION}

The taxonomy of the genus Arachniodes Blume in North India is intensively studied last several years. The main aim of the study is to provide a comprehensive list of the genera of Dryopteridaceae grow in North-East India and to enumerate the species of Arachniodes of the region along with their detailed description and colorful photographs. Based on literature review stated that no much work is paid on cytology, and molecular identity of the genus. Hence it is necessary to study the Cytology, Palynology and molecular studies of this genus from India and that is an urgent need for understang the taxon in a better way.

\section{Acknowledgements}

The first author is thankful to Director, Botanical Survey of India, Kolkata for his support and encouragements and also thankful to C.R.F. Jenkins, Nepal for his support and encouragement and also thankful to Department of Science and Technology, New Delhi for giving financial Support through DST SERB Scheme.

\section{LITERATURE CITED}

Beddome, R.H. 1876. Supplements to the Ferns of Southern India and British India, etc. Gantz. Brothers, Madras.

Beddome, R.H. 1892. Handbook to the ferns of British India, Ceylon and the Malaya Peninsula. Thacker Sprink and Company, Calcutta. 
Beddome, R. H. 1865 - 1870. The Ferns of British India. 1-120 (1865), 121-150 (1866); 2: 151-210 (1866), 211-255(1867); 256-300 (1868), 301-330(1869), 331-345(1870). Ghant Brothers Madras. Reprinted in combined Volume.

Beddome, R.H. 1883. A Handbook to the Ferns of British India, Ceylon and Malay Peninsula. Thackar Spink \& Co, Calcutta [reprint ed. 1978, Today and Tomorrows Printers and Publishers, New Delhi.]

Biswas, K, P. 1941. The flora of the Aka Hills, Indian For. Rec. 3 (1): 1 - 62.

Burkill, I. H. 1924. The Botany of the Abor Expedition, Rec. Bot. Surv. India 10(1): 1 - 154; $10(2): 155-420$.

Chandra, P. 1980. Botanical Exploration in Tawang, Ferns and Fern Allies, N.B.R.I. Res. Publ. no. 73 (N.S.). Nova Hedwigia 32: 399 - 414.

Chandra, S. 2000. The ferns of India (Enumeration, Synonyms \& Distribution), International Book Distributors, Dehra Dun.

Chapman, A.D. 2006. Numbers of Living Species in Australia and the World. Report for the Department of the Environment and Heritage, Canberra, Australia.

Chauhan, A.S. \& Wadhwa, B.M. 1996. Pteridophytes, In: Hajra, P. K. (ed.), A Contribution to the Flora of Namdapha, Arunachal Pradesh. Botanical Survey of India, Calcutta.

Clarke, C.B. 1880. A review of the ferns of northern India etc., Trans. Linn. Soc. (Lond.), ser. 2, Bot. 1: $425-619$.

Clarke, C.B. \& Baker, J.G. 1888. Supplementary note on the Ferns of Northern India. $J$. Linn. Soc. London.

Deb, D.B. \& Dutta, R.M. 1972. Contribution to the flora of Tirap Frontier Division, J. Bombay Nat. Hist. Soc. 68 (3): 573 - 595.

Fisher, C.E.C. 1938. The flora of the Lushai Hills. Bot. Surv. India 12: 75-161.

Fraser-Jenkins, C.R. 2010. A brief comparison of modern Pteridophyte classifications (families and genera in India). Indian Fern J. 26: 107 - 131.

Griffith, W. 1845. On Azolla and Salvinia. Calcutta J. Nat. Hist. 5: 227.

Hooker, W.J. 1846 - 1864. Species Filicum, Volumes I - V. [1: 1 - 245 t.1 - 70 (1846); 2: $1-250$, t. $71-149$ (1858); 3: $1-291$ t. $141-210$ (1860); 4: $1-292$, t. $211-280$ (1862); 5: 1 - 314,t. 281 - 303 (1864)]. Lubrecht \& Cramer, London.

Hooker, W.J. \& Baker, J.G. 1864. Synopsis Filicum, or A Synopsis of All Known Ferns, including the Osmundaceae, Schizeaceae, Marattiaceae, and Ophioglossaceae. Robert Hardwicke, London.

Kachroo, P. 1975. Fern flora of Assam with some Phytogeographical notes. J. Indian Bot. Soc. 54: $13-26$.

Kramer, K.U. 1990. Dryopteridaceae. In: Kramer, K.U.; Green, P.S. (eds.), The Families and Genera of Vascular Plants. Vol. 1. Pteridophytes and Gymnosperms. SpringerVerlag, Berlin. Pp. 101 - 144.

Panigraghi, G. \& Chowdhury, S. 1962 Enumeration and distribution of fern allies in Eastern India. Proc 49th Ind. Sci. Congr. Cuttack (Abs). Pp. 255 - 256.

Panigraghi, G. \& Patnaik, S.N. 1961. Enumeration of correct nomenclature of polypodiaceae in Eastern India, Indian For. 87(4): 242. 
Panigraghi, G. 1962. Pteridophytes of the Eastern India-1. Enumeration of the species collected and their nomenclature. Bull. Bot. Surv. India 2(3 \& 4): 309 - 314.

Pichi Sermolli, R.E.G. 1977. Tentamen Pteridophytorum genera in taxonomicum ordinem redigendi Webbia 31(2): 313 - 512 .

Proctor, J.; Haridasan K. \& Smith, G.W. 1998. How far does lowland evergreen tropical rainforest go? Global Ecol. Biogeo. Lett. 7: 141 - 146.

Rao, A.S. \& Hajra, P.K. 1980. Fern allies and ferns of Kameng District, Arunachal Pradesh Indian For. 106 (5): 327 - 349.

Rawat, V.K.; Sahu, T.R. \& Dixit, R.D. 2005. A preliminary account of the pteridophytes from Mehao Wildlife Sanctuary, Arunachal Pradesh, India, J. Econ. Tax. Bot. 29(4): $738-742$.

Rothwell, G.W. \& Stockey, R.A. 2008. Phylogeny and evolution of ferns: a paleontological perspective. Chapter 13. pp. 334-368. In. Ranker, T.A. \& Haufler, C.H. (eds.), Biology and evolution of ferns and lycophytes. Cambridge Univ. Press.

Roxburgh, W. 1844. The Cryptogamous plants. Cal. J. Nat. Hist. $4-476$.

Roxburgh, W. 1844. Flora Indica: The Cryptogamous Plants of Dr. Roxburgh, Forming the Fourth and Last Part of the Flora Indica. Cal. J. Nat. Hist. Miscel. Arts Sci. India 4: $463-520$.

Singh, S. \& Panigrahi, G. 2005. Ferns and Fern-Allies of Arunachal Pradesh, Vols. 1 \& 2. Bishen Singh Mahendra Pal Singh, Dehra Dun.

Smith, A.R.; Pryer, K.; Schuettpelz, E.; Korall, P.; Schneider, H. \& Wolf, P.J. 2006. A classification for extant ferns. Taxon 55: $705-731$.

Srinivasan, K.S. 1959. Report on a botanical tour to Bomdila, N.E.F.A. (May, 1955), Rec. Bot. Surv. India 17(2): 1 - 37.

Vasudeva, S.M.; Bir, S.S. \& Kachroo, P. 1990. Pteridophytic flora of North-Eastern India 3 (Families: Aspleniaceae-Oleandraceae), Indian Fern J. 7(1-2): 66 - 85.

Widen, C.J.; Fraser-Jenkins, C.R.; Reichstein, T.; Gibby, M. \& Sarela, J. 1996. Phloroglucinol derivatives in Dryopteris section Fibrillosae and related taxa (Pteridophyta, Dryopteridaceae). Ann. Bot. Fennici 33: 69 - 100. 